\title{
Measurement of photon-pair generation in waveguide arrays with specialized poling
}

\author{
Francesco Lenzini $^{1,2}$, James Titchener ${ }^{3}$, Paul Fisher ${ }^{1,2}$, Andreas Boes ${ }^{4}$, \\ Alexander N. Poddubny ${ }^{5,6,3}$, Sachin Kasture ${ }^{1,2}$, Ben Haylock ${ }^{1,2}$, Matteo Villa ${ }^{1,2}$, \\ Arnan Mitchell ${ }^{4}$, Alexander S. Solntsev ${ }^{3}$, Andrey A. Sukhorukov ${ }^{3}$, and Mirko Lobino ${ }^{1,2}$ \\ 1) Centre For Quantum Dynamics, Griffith University, Brisbane QLD 4111, Australia \\ 2) Queensland Micro- and Nanotechnology Centre, Griffith University, Brisbane QLD 4111, Australia \\ 3) ARC Centre of Excellence CUDOS, Nonlinear Physics Centre, Research School of Physics and Engineering, \\ The Australian National University, Canberra ACT 2601, Australia \\ 4) ARC Centre of Excellence CUDOS, School of Engineering, RMIT University, Melbourne VIC 30000, Australia \\ 5) ITMO University, Saint Petersburg 197101, Russia \\ 6) Ioffe Physical-Technical Institute of the Russian Academy of Science, Saint Petersburg 194021, Russia \\ francesco.lenzini@griffithuni.edu.au
}

\begin{abstract}
We present the realization of an inhomogeneously poled nonlinear waveguide array for the generation of photon pairs. The device is characterized by coincidence counting and a novel method based on reversed sum-frequency generation measurements.
\end{abstract}

OCIS codes: $270.5585,190.4390$

\section{Introduction}

Integrated waveguide circuits offer a stable and scalable platform for the implementation of Quantum Logic and Communication optical networks. Such circuits usually employ a first stage for the generation of single photons followed by a linear network of directional couplers for their subsequent manipulation. This standard configuration can be replaced by the use of an array of quadratic nonlinear waveguides, allowing for the simultaneous generation of photon pairs by Spontaneous Parametric Down-Conversion (SPDC) and their manipulation via the evanescent coupling with adjacent waveguides [1]. Besides offering a substantial improvement in scalability, this scheme can employ novel ways to control the quantum interference of the generated photon pairs. In particular, the use of specially designed periodic poling patterns can allow to control the bunching and anti-bunching of photon pairs at the output of the array in order to generate useful quantum resource states, such as Bell States in path encoding [2].

As a first experimental demonstration of this concept, we present the realization of a three-waveguide array for the generation of photon pairs with integrated pump filtering (Fig. 1a). A $2 \mathrm{~cm}$ long periodically poled waveguide is used for the generation of signal and idler photon pairs via SPDC. At the output of the device signal and idler photons are separated to waveguides 2 and 3 with one photon in each waveguide and filtered from the pump beam, which remains confined to waveguide 1 . This separation is achieved by the use of a specially designed periodic poling pattern with defects at 5 different locations consisting in translations of the poling structure by half a period. The introduced translations are used to induce a $\pi$ phase shift on wavefunctions generated at different locations of the array, resulting in a controlled constructive interference for the output state only when signal and idler photons are separated to the desired waveguides.

\section{Experimental results}

The waveguide array is fabricated on a periodically poled z-cut Lithium Niobate wafer by the use of standard photolithography and an Annealed-Reverse Proton Exchange technique [3]. We characterize the biphoton wavefunction produced from the device by exploiting the analogy between SPDC and classical nonlinear processes. While previous realizations have a validity limited to a single and lossless waveguide [4,5], we extend this approach to complex poled structures by establishing a rigorous analogy with the reverse process of SPDC, i.e. Sum-Frequency Generation (SFG). Fig. 1b shows the proposed scheme for the SFG characterization: signal and idler beams are injected in waveguides $n_{s}, n_{i}$ in the opposite direction of the array. The sum-frequency field generated at the input of the first waveguide gives direct information on the wavefunction element $\Psi_{n_{s} n_{i}}$. 
We first reconstruct the phase-matching function of the target state by scanning the frequencies of signal and idler beams injected in waveguides 2-3 and measuring the corresponding SFG efficiencies (Fig. 1c). We then select the diagonal with maximum conversion efficiency $\left(\lambda_{s}^{-1}+\lambda_{i}^{-1}=775^{-1} \mathrm{~nm}^{-1}\right)$ and reconstruct the elements of the biphoton wavefunction by measuring the SFG efficiencies for all the other permutations of signal-idler beams (Fig. 1d). The presence of non-zero elements with signal-idler in waveguides 2-1, as well the asymmetry in the reconstructed phasematching function, are likely due to non-uniformities in the refractive index profile of the periodically poled waveguide. Nevertheless, we observe the key result that the wavefunction corresponding to the output state (for $n_{s}, n_{i}=2,3$ ) is very close to the designed anti-correlated state.

SPDC measurements are finally performed by injecting a pump beam with wavelength $\lambda_{p}=775 \mathrm{~nm}$ in waveguide 1 and measuring the photon coincidences between each pair of waveguides with two InGaAs avalanche photodiodes and a time-to-digital converter. In Fig. 1e we report the biphoton wavefunction reconstructed for a pump power $P_{p}=$ $15 \mu \mathrm{W}$, showing an excellent agreement with the SFG characterization.

(a)

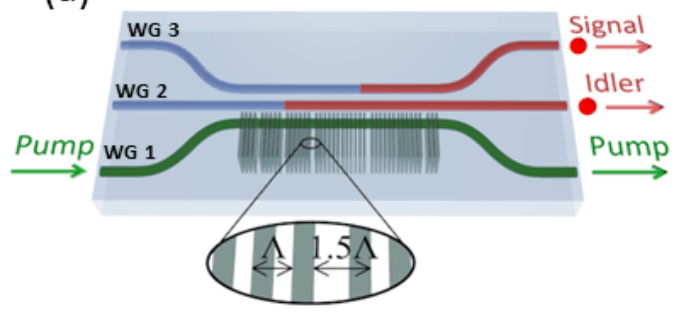

(b)

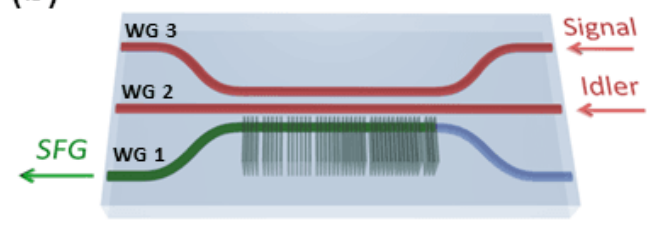

(c)

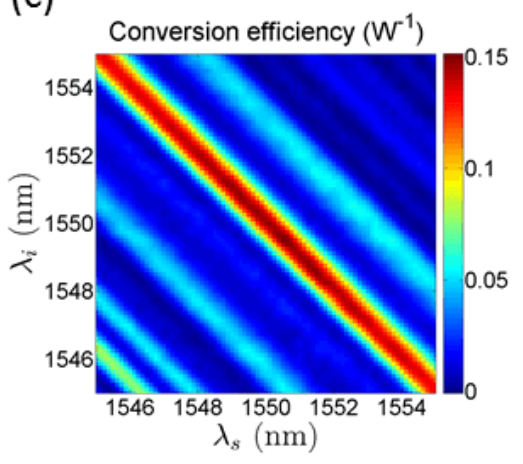

(d)

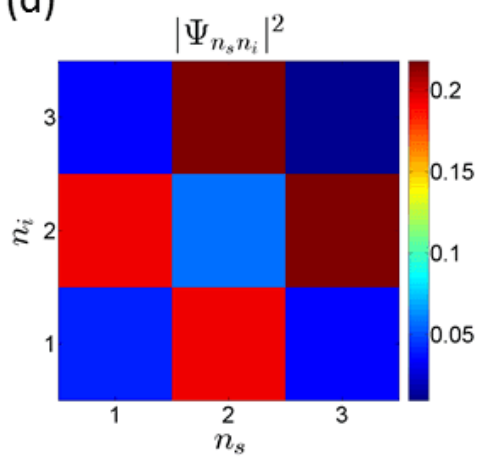

(e)

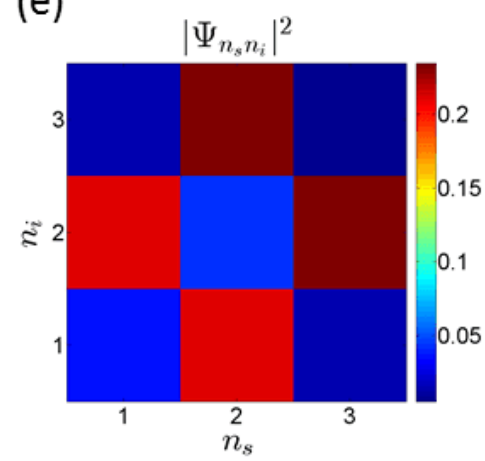

Fig. 1. (a) Schematic of the waveguide array and the periodic poling pattern. (b) Proposed scheme for the SFG characterization. (c) Phase-matching function of the biphoton state reconstructed by SFG measurements with signal-idler in waveguides 2-3. (d) Biphoton wavefunction produced from the device reconstructed with the SFG characterization. (e) Biphoton wavefunction produced from the device reconstructed by SPDC measurements.

In conclusion, we have experimentally shown the first realization of a nonlinear waveguide array with specially designed periodic poling pattern for the generation of specific quantum states, as well a novel method for the classical characterization of the SPDC wavefunction produced from complex waveguide structures. These results represent a significant step towards the realization of waveguide arrays for the generation of all-optically reconfigurable entangled states and are of practical importance for the characterization of on-chip single photon source fabrication.

\section{References}

1. A. S. Solntsev et al., Phys. Rev. X 4, 031007 (2014).

2. J. Tichener et al., Phys. Rev. A 92, 033819 (2015).

3. F. Lenzini et al., Opt. Exp. 23, 1748-1756 (2015).

4. A. Eckstein et al., Laser Photon. Rev. 8, L76-L80 (2014).

5. L. G. Helt, M. J. Steel, Opt. Lett. 40, 1460-1463 (2015). 